As expected there was a correlation between arousal at the onset of sampling and total duration of crying. It is often noted by technicians taking regular samples from infants that those who are still or asleep often cry less in response to sampling than infants who are active or 'fussing'.
1 Smith BA, Fillion TJ, Blass EM. Orally mediated sources of calming in 1 to 3 day old human infants. Developmental Psychology 1990; 26: 731-7.

2 Blass EM, Hoffmeyer LB. Sucrose as an analgesic for newborn infants. Pediatrics 1991; 87: 215-8.

3 Blass EM, Fitzgerald E, Kehoe P. Interactions between sucrose, pain and isolation distress. Pharmacology sucrose, pain and isolation distress.
Biochemistry and Behaviour 1987; 26: 483-9.

4 Gladman G, Chiswick ML. Skin conductance and arousal in the newborn. Arch Dis Child 1990; 65: 1063-6.

\title{
Neonatal cerebral venous thrombosis
}

Have you ever wondered what it is that makes people send their papers to one journal rather than another? Presumably a major factor is their judgment as regards likely success, but surely the predicted readership is important. Take Annals of Neurology, for instance. Now, I would guess that the average reader of that splendid journal would be hard pressed to choose between a paper on neonatal neurology and the latest revision of the Ulan Bator and district public bus timetable as regards relevance to his practice. Similarly, most neonatologists will consult the journal at a frequency approximating to that with which an Englishman wins the Wimbledon singles championship. Nevertheless, the July 1992 issue contains a paper of considerable relevance to paediatricians.

Reports of neonatal cerebral venous thrombosis involving either the dural sinuses or deeper veins are uncommon and have usually described a very small number of babies with thrombosis either idiopathic or secondary to conditions such as severe birth asphyxia, polycythaemia, dehydration, sepsis, or protein C deficiency. A report in 1988 described 17 neonates with dural sinus thrombosis detected on computed tomography over a period of eight years. ${ }^{1}$ Now workers in Boston have used both computed tomography and magnetic resonance imaging to show cerebral venous thrombosis in seven newborn infants seen at the Floating Hospital for Infants and Children over a period of only three months (Michael J Rivkin and colleagues, Annals of Neurology 1992; 32: 51-6).

Six of the seven were born at term and the other at 32 weeks' gestation. Three presented with a single focal seizure and four with lethargy. Computed tomography showed evidence of dural sinus thrombosis in six babies and of frontal lobe hypoplasia in the seventh. Magnetic resonance imaging identified venous thrombosis in all seven and in four babies it showed more extensive venous thrombosis than had been seen on the computed tomogram. In addition to standard magnetic resonance images, phase imaging was used and this technique, which is able to demonstrate blood flow in cerebral veins, showed obstruction to flow in all seven patients.

The condition appears to be relatively benign as none of the babies had recurrent seizures and the only one to be neurologically abnormal at follow up was the baby with hypoplastic frontal lobes. The thrombosis was designated idiopathic in all seven and no baby was given anticoagulant or thrombolytic treatment. It seems possible that the condition is more common than we realise. 